\title{
Cell death in a co-culture of hepatocellular carcinoma cells and human umbilical vascular endothelial cells in a medium lacking glucose and arginine
}

\author{
MINORU TOMIZAWA ${ }^{1}$, FUMINOBU SHINOZAKI ${ }^{2}$, YASUFUMI MOTOYOSHI ${ }^{3}$, \\ TAKAO SUGIYAMA $^{4}$, SHIGENORI YAMAMOTO 5 and NAOKI ISHIGE ${ }^{6}$ \\ Departments of ${ }^{1}$ Gastroenterology, ${ }^{2}$ Radiology, ${ }^{3}$ Neurosurgery, ${ }^{4}$ Rheumatology, ${ }^{5}$ Pediatrics and ${ }^{6}$ Internal Medicine, \\ National Hospital Organization Shimoshizu Hospital, Yotsukaido, Chiba 284-0003, Japan
}

Received March 19, 2015; Accepted May 18, 2016

DOI: $10.3892 / \mathrm{ol} .2016 .5454$

\begin{abstract}
Human primary hepatocytes are able to survive in a medium without glucose and arginine that is instead supplemented with galactose and ornithine (hepatocyte selection medium; HSM). This is because the cells produce glucose and arginine by the action of galactokinase (GALK) and ornithine carbamoyltransferase (OTC), respectively. It was expected that hepatocellular carcinoma (HCC) cells do not survive in HSM. In the current study, HCC cell lines (namely HLE, HLF, PLC/PRL/5, Hep3B and HepG2) and human umbilical vascular endothelial cells (HUVECs) were cultured in HSM, and the expression levels of GALK1, GALK2 and OTC were analyzed by reverse transcription-quantitative polymerase chain reaction. HLE, HLF and PLC/PRL/5 cells died on day 11, while Hep3B, HepG2 and HUVECs died on day 7. HLF cells were further analyzed as these cells had lower expression levels of GALK1, GALK2 and OTC compared with adult liver cells, and survived until day 11. In these cells, the expression levels of GALK1, GALK2 and OTC did not change on days 3 and 7 as compared to day 0 . In addition, a co-culture of HLF cells with HUVECs was established and the medium was changed to HSM. It was observed that HLF cells and HUVECs in co-culture were damaged in HSM. In summary, HCC cells and HUVECs died in a medium without glucose and arginine that was supplemented with galactose and ornithine. HCC cells and HUVECs were damaged in HSM, suggesting a potential application for treatment with the medium.
\end{abstract}

Correspondence to: Dr Minoru Tomizawa, Department of Gastroenterology, National Hospital Organization Shimoshizu Hospital, 934-5 Shikawatashi, Yotsukaido, Chiba 284-0003, Japan E-mail: nihminor-cib@umin.ac.jp

Key words: galactokinase, ornithine carbamoyltransferase, gluconeogenesis, urea cycle

\section{Introduction}

Hepatocellular carcinoma (HCC) is a common malignancy worldwide and its prognosis is poor (1). HCC tissue is composed of tumor cells and blood vessels (2). Treatment of this disease includes local ablation, chemoembolization and systemic administration of chemotherapeutic agents $(3,4)$; however, this is limited by hepatotoxicity (5).

Glucose and pyruvate are important sources of energy for cell survival $(6,7)$. Galactose enters glycolysis as a substrate for galactokinase (GALK), which is expressed in the liver and kidney $(8,9)$. Therefore, hepatocytes are expected to survive in a galactose-containing medium, in the absence of glucose or pyruvate $(10,11)$.

Arginine, an essential amino acid, is produced from ornithine by the action of ornithine carbamoyltransferase (OTC) in the urea cycle (12). As the urea cycle is exclusive to hepatocytes, these cells are able to survive in a medium lacking glucose and arginine that is supplemented with galactose and ornithine (13).

We previously developed a hepatocyte selection medium (HSM) that lacks glucose and arginine but contains galactose and ornithine (14). This medium was demonstrated to purify primary human hepatocytes from co-culture with human induced pluripotent stem cells (15). In the same manner, the current study attempted to eliminate HCC cell lines using HSM. Additionally, a co-culture of HCC cells with human umbilical vascular endothelial cells (HUVECs) was studied as a model of HCC tissues.

\section{Materials and methods}

Cell lines and culture media. The human HCC cell lines HLE, HLF, PLC/PRL/5, Hep3B and HepG2 were purchased from the Riken Bioresource Centre Cell Bank (Tsukuba, Japan). Hepatoma cells were cultured in Dulbecco's modified Eagle's medium (DMEM; Sigma-Aldrich, St. Louis, MO, USA), and supplemented with $10 \%$ fetal bovine serum (Thermo Fisher Scientific, Inc., Carlsbad, CA, USA). HUVECs and their culture medium, Endothelial Cell Growth Media BulletKit ${ }^{\mathrm{TM}}$ (EGM), were purchased from Lonza Group Ltd. (Walkersville, MD, USA). Cell lines were cultured with $5 \%$ carbon 
Table I. List of primers used for polymerase chain reaction, GenBank accession numbers and expected product lengths.

\begin{tabular}{|c|c|c|c|}
\hline Primer & Sequence $\left(5^{\prime}-3^{\prime}\right)$ & GenBank accession number & Product size (bp) \\
\hline RPL19 forward & CGAATGCCAGAGAAGGTCAC & ВC000530 & 157 \\
\hline RPL19 reverse & CCATGAGAATCCGCTTGTTT & & \\
\hline Albumin forward & CCATGAGAATCCGCTTGTTT & NM_000477 & 114 \\
\hline Albumin reverse & GCAAAGCAGGTCTCCTTATCGTC & & \\
\hline GALK 1 forward & GCTGCTTGAGAGAGGTAGAAGGTG & NM_000154 & 153 \\
\hline GALK1 reverse & TCACGACTTACTGGAGCAGGATG & & \\
\hline GALK2 forward & TCACGACTTACTGGAGCAGGATG & NM_002044 & 177 \\
\hline GALK2 reverse & CAAAACCAAAGCCCCACCTC & & \\
\hline OTC forward & GGACATTTTTACACTGCTTGCCC & BC 107153 & 105 \\
\hline OTC reverse & TCCACTTTCTGTTTTCTGCCTCTG & & \\
\hline PEPCK forward & GGCTACAACTTCGGCAAATACCTG & NM_002591 & 167 \\
\hline PEPCK reverse & TTGAACATCCACTCCAGCACCCTG & & \\
\hline G6P forward & AACAGAGCCAGTCACAGCACCAAG & NM_000151 & 139 \\
\hline G6P reverse & CCTCAGGAAATCCATTGATACGG & & \\
\hline Cyclin D1 forward & AGAGGCGGAGGAGAACAAACAG & NM_053056 & 180 \\
\hline Cyclin D1 reverse & AGGCGGTAGTAGGACAGGAAGTTG & & \\
\hline
\end{tabular}

RPL19, ribosomal protein L19; GALK, galactokinase; OTC, ornithine carbamoyltransferase; PEPCK, phosphoenolpyruvate carboxykinase; G6P, glucose-6-phosphatase.

dioxide at $37^{\circ} \mathrm{C}$ in a humidified chamber, and were observed using a CKX41N-31PHP microscope (Olympus Corporation, Tokyo, Japan).

Co-culture of HLF or PLC/PRL/5 with HUVECs. HUVECs were seeded onto a 24-well plate coated with $150 \mu$ l Matrigel (BD Biosciences, Franklin Lakes, NJ, USA) per well, at a density of $1.0 \times 10^{4}$ cells/well. After one day of culture in EGM, the medium was discarded and the HLF cells were added at a density of $1.0 \times 10^{4}$ cells/well. The cells were cultured in DMEM supplemented with $10 \%$ fetal bovine serum.

Preparation of HSM. HSM was prepared from amino acid powders following the formulation of Leibovitz's L-15 medium (Thermo Fisher Scientific, Inc.), excluding arginine, tyrosine, glucose and sodium pyruvate, but with the addition of galactose $(900 \mathrm{mg} / \mathrm{l})$, ornithine $(1 \mathrm{mM})$, glycerol $(5 \mathrm{mM})$ and proline (260 mM), all obtained from Wako Pure Chemical Industries, Ltd. (Osaka, Japan) (15). Proline (30 mg/l) was added as it is essential for DNA synthesis (16). KnockOut Serum Replacement (KSR) (Thermo Fisher Scientific, Inc.) was added at a final concentration of $10 \%$, and was used instead of fetal calf serum (FCS) to establish defined xeno-free conditions. Depending on the experiment, KSR was dialyzed against phosphate-buffered saline (PBS) to remove the amino acids and glucose.

Reverse transcription-quantitative polymerase chain reaction $(R T-q P C R)$. Cells were seeded in 6-well plates (Asahi Techno Glass, Tokyo, Japan) and cultured in DMEM supplemented with $10 \%$ fetal bovine serum. When the cultured cells reached $70 \%$ confluency, the medium was changed to HSM. At $48 \mathrm{~h}$ after the media was changed to HSM, total RNA (5 $\mu \mathrm{g})$ was isolated using Isogen (Nippon Gene, Co., Ltd., Tokyo, Japan).
cDNA was synthesized using SuperScript ${ }^{\circledR}$ III Reverse Transcriptase and oligo dT primers, as per the manufacturer's instructions (Thermo Fisher Scientific, Inc.). Human fetal liver total RNA and human adult liver total RNA were purchased from Clontech Laboratories Inc. (Mountain View, CA, USA). The PCR primers, GenBank accession numbers and expected product sizes are listed in Table I. RT-qPCR was conducted with $25 \mu \mathrm{l}$ of Fast SYBR Green Master Mix (Thermo Fisher Scientific, Inc.) in total volume of $50 \mu \mathrm{l}$ for each reaction in the Mini Opticon system (Bio Rad Laboratories, Inc., Hercules, CA, USA). PCR was performed for 40 cycles, with denaturation $\left(95^{\circ} \mathrm{C}\right)$ and annealing-extension $\left(60^{\circ} \mathrm{C}\right)$ times of $5 \mathrm{sec}$ each. Ribosomal protein L19 was used as an internal control. Expression levels of the genes involved were automatically analyzed with this system. RPL19 was used as an endogenous control to monitor the amount of mRNA, as it is constitutively expressed housekeeping gene (17). The gene expression levels were analyzed automatically using the Mini Opticon system based on the delta-delta cycle threshold (ddCt) method (18). The relative expression was calculated as the expression level of a specific gene divided by that of RPL19.

Statistical analysis. One-way analysis of variance was performed with JMP 10.0.2 software (SAS Institute, Cary, $\mathrm{NC}$, USA). $\mathrm{P}<0.05$ was considered to indicate a statistically significant difference.

\section{Results}

GALK1, GALK2 and OTC expression in HCC cells. To observe the expression levels of GALK1 (Fig. 1A), GALK2 (Fig. 1B) and OTC (Fig. 1C) in each HCC cell line, RT-qPCR was performed. This revealed that expression levels of GALK1 and 

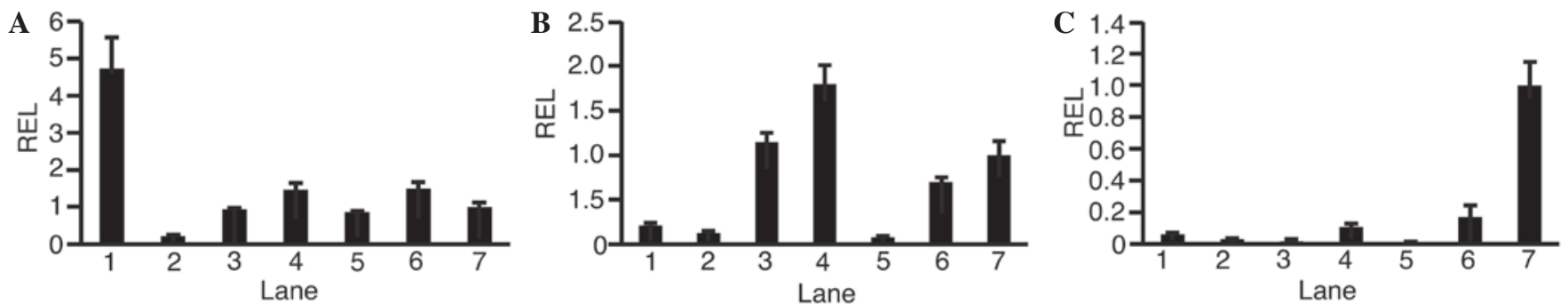

Figure 1. Expression of galactokinase 1, galactokinase 2 and ornithine carbamoyltransferase in various hepatocellular carcinoma and normal liver cell lines. Expression levels of (A) galactokinase 1, (B) galactokinase 2 and (C) ornithine carbamoyltransferase were analyzed by reverse transcription-quantitative polymerase chain reaction. Lanes: $1 \mathrm{HLE} ; 2$, HLF; 3, PLC/PRL/5; 4, Hep3B; 5, HepG2; 6, human fetal liver; 7, human adult liver. Graphs represent the relative expression levels (REL) normalized against that of adult liver. Error bars indicate standard deviation; $\mathrm{n}=3$.

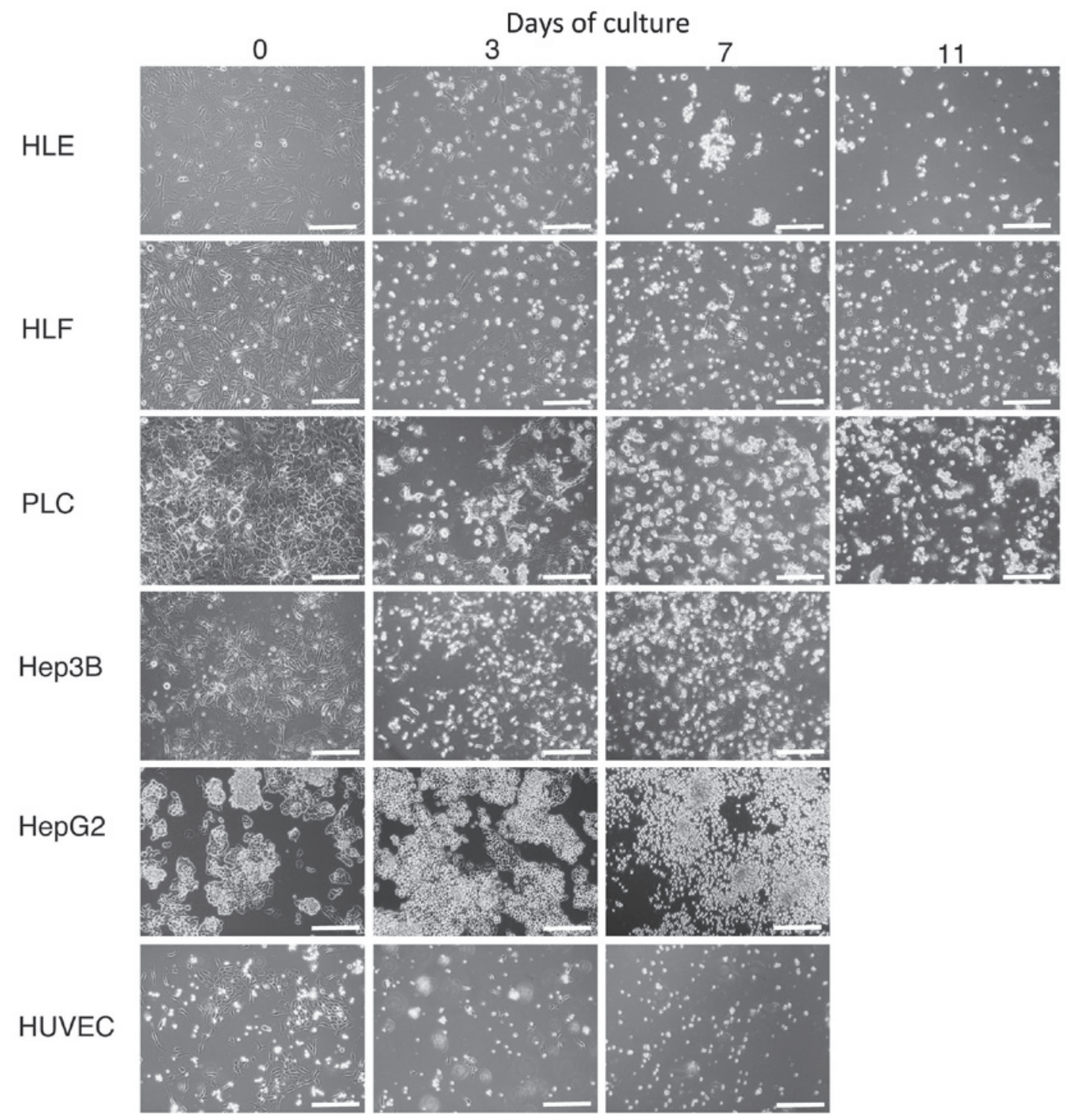

Figure 2. Hepatoma cells, HLE, HLF, PLC/PRL/5, Hep3B, HepG2 and HUVECs were cultured in hepatocyte selection medium and observed by microscopy. A small number of HLE, HLF and PLC/PRL/5 cells survived on day 7. On day 11, HLE, HLF and PLC/PRL/5 cells died. Original magnification, x100; scale bar, $50 \mu \mathrm{m}$. HUVEC, human umbilical vascular endothelial cell.

GALK2 varied depending on the type of cell. Expression levels of OTC in HCC cells were lower compared with in adult liver cells. In HLF cells, expression levels of GALK1 $(0.22 \pm 0.03$; $\mathrm{P}<0.01)$, GALK2 $(0.12 \pm 0.03 ; \mathrm{P}<0.01)$ and OTC $(0.03 \pm 0.00$; $\mathrm{P}<0.01)$ were lower compared with in adult liver cells.
Effect of HSM on HCC cells and HUVECs. To address the possibility that HCC cells and HUVECs would undergo cell death in HSM, cultures were established (Fig. 2). Hep3B, HepG2 and HUVECs were microscopically observed to have died after being cultured for 7 days in HSM, which is 

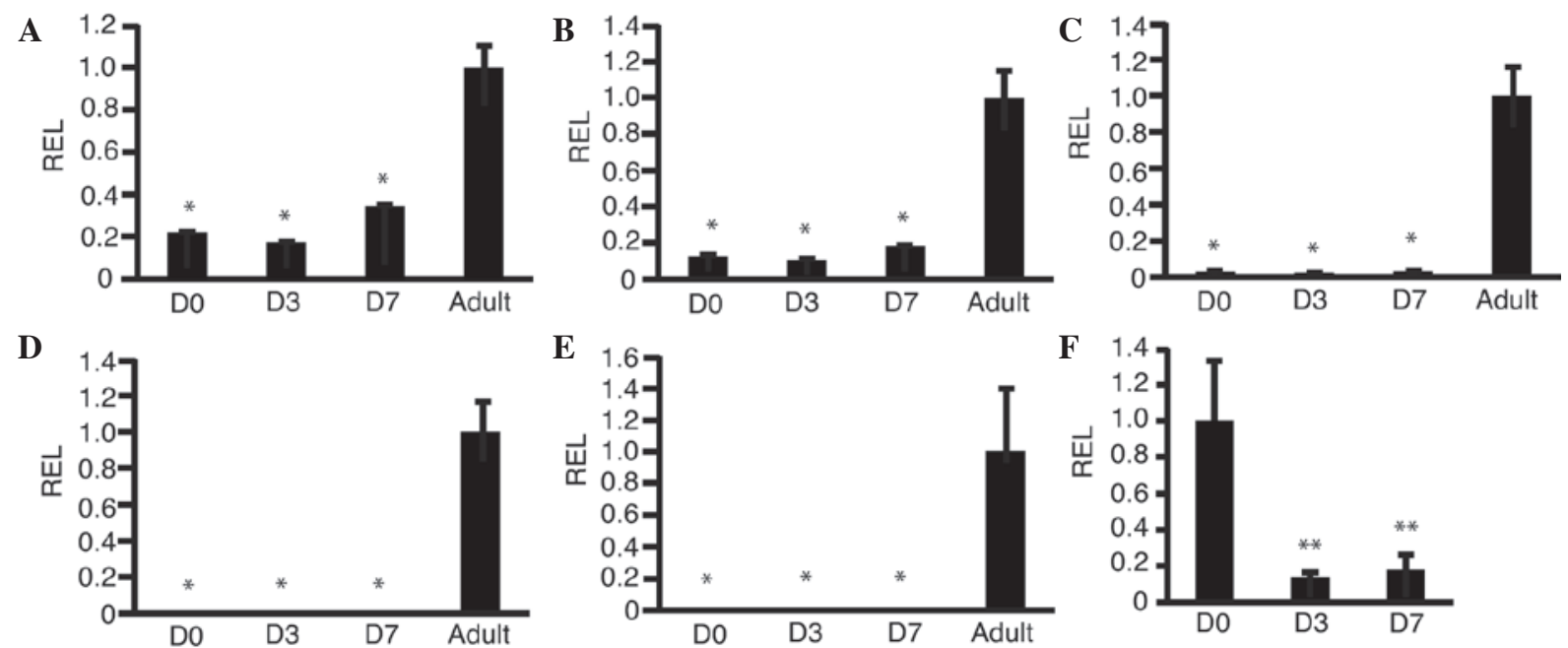

Figure 3. Changes in expression levels of genes involved in metabolism and cyclin D1. HLF cells were cultured in HSM and RNA was isolated. Reverse transcription-quantitative polymerase chain reaction was performed. Graphs show the REL, normalized against human adult liver cells, of (A) galactokinase 1, (B) galactokinase 2, (C) ornithine carbamoyltransferase, (D) phosphoenolpyruvate carboxykinase, (E) glucose-6-phosphatase and (F) cyclin D1; "P $<0.05$ as compared with adult, ${ }^{* *} \mathrm{P}<0.05$ as compared with D0 $(n=3)$. D0, day 0 after culture in HSM; D3, three days after culture in HSM; D7, seven days after culture in HSM; adult, human adult liver; HSM, hepatocyte selection medium; REL, relative expression levels.

A

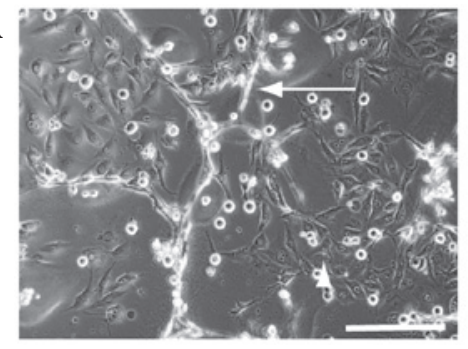

B

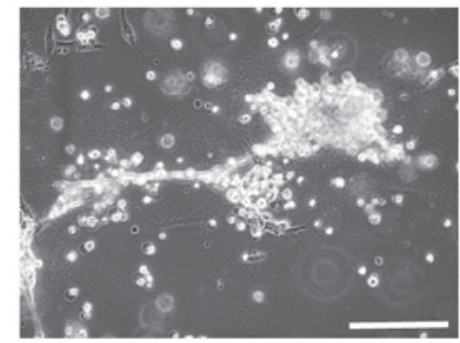

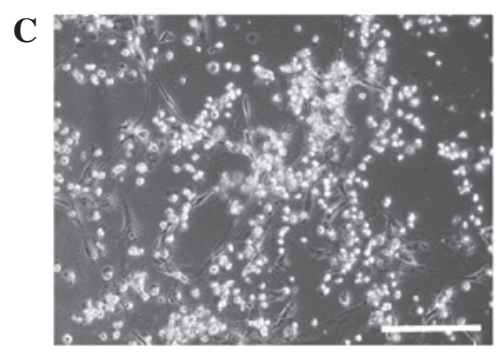

Figure 4. (A) Co-culture of HLF with HUVECs was established as an in vitro model of hepatoma tissues. Cells were observed under the microscope at (B) $18 \mathrm{~h}$ and (C) $60 \mathrm{~h}$ after the medium was changed to hepatocyte selection medium. Two cells started to die at $18 \mathrm{~h}$. Almost all the cells died at $60 \mathrm{~h}$. Original magnification, x100; scale bar, $50 \mu \mathrm{m}$; arrow, HUVECs; arrowhead, HLF. HUVECs, human umbilical vascular endothelial cells.

morphologically shown in Fig. 2. A small number of HLE, HLF and PLC/PRL/5 cells survived on day 7. On day 11, all HLE, HLF and PLC/PRL/5 cells died, as observed morphologically

Expression of metabolic genes and cyclin D1 in HLF cells. The HLF cell line was selected for further analysis of the change in expression levels of metabolic genes as its expression levels of GALK1, GALK2 and OTC were lower compared with those in adult liver; however, it was hypothesized that certain genes involved in metabolism would be upregulated to promote survival in HSM. Cyclin D1 expression was also analyzed as this gene is involved in cell proliferation; it was hypothesized that cell proliferation would be suppressed in HSM. RT-qPCR was performed to investigate changes in expression levels of GALK1 (Fig. 3A), GALK2 (Fig. 3B), OTC (Fig. 3C), phosphoenolpyruvate carboxykinase (PEPCK) (Fig. 3D), glucose-6-phosphatase (G6P) (Fig. 3E) and cyclin D1 (Fig. 3F). The expression levels of GALK1 changed from $0.21 \pm 0.04$ (day 0) to $0.33 \pm 0.05$ (day 7) $(\mathrm{P}=0.01)$. The expression levels of GALK2 changed from $0.11 \pm 0.03$ (day 0 ) to $0.18 \pm 0.02$ (day 7) $(\mathrm{P}=0.03)$. The expression levels of OTC changed from $0.06 \pm 0.01$ (day 0 ) to $0.07 \pm 0.02$ (day 7) $(\mathrm{P}=0.67$ ). The expression levels of PEPCK and G6P were $0.00 \pm 0.00$ on day 0 and day 7 . These data suggested that GALK1 and GALK2 were upregulated in HSM. The expression levels of cyclin D1 changed from $1.00 \pm 0.33$ to $0.18 \pm 0.09$ ( $\mathrm{P}<0.01)$. These results suggested that cyclin D1 was downregulated as predicted.

Effect of HSM on HUVEC and HCC co-culture. Co-culture of HLF cells with HUVECs was established as an in vitro model of HCC tissues (Fig. 4A). HLF cells and HUVECs were cultured in HSM and observed by microscopy. HLF and HUVECs began to die at $18 \mathrm{~h}$ (Fig. 4B), and almost all the cells had died after $60 \mathrm{~h}$ (Fig. 4C) of culture.

\section{Discussion}

Cellular energy deprivation is a potential treatment for HCC (19). For example, HCC cells do not survive ischemia, which causes deprivation of glucose (20). However, such a lack of glucose and oxygen also may damage normal hepatocytes. To treat HCC with the deprivation of glucose, survival of normal hepatocytes would be a major problem.

HCC cells can modify their mechanism to survive under the deprivation of glucose (21). These literatures suggest that HCC cells may not die without glucose alone. The HSM used 
in the present study did not contain arginine or glucose. It was expected that HSM would be more effective for death of HCC cells compared with glucose deprivation alone.

Primary human hepatocytes are able to survive in HSM (15). In the present study, all HCC cells and HUVECs died within 7 or 11 days of culture. HSM also caused the death of HLF cells and HUVECs in co-culture. The co-culture of HLF and HUVECs could be used as a model for HCC tissues, as HUVECs were used as a feeding artery. It was expected that the feeding artery of HCC would also be damaged with HSM. If HCC cells survived in HSM, the cells would not survive as the feeding artery was damaged, and glucose and oxygen were not supplied. Death of the two cell types in HSM indicated that HSM was applicable for the treatment of HCC. Notably, normal hepatocytes survive in HSM. These results suggested that HSM would be useful in the treatment of HCC tissues, and safe for normal hepatocytes.

Arginine is synthesized from citrulline catalyzed by argininosuccinate synthetase (22). Arginine deprivation is, therefore, a treatment for a type of HCC that lacks argininosuccinate synthetase (22). HSM would not be harmful to normal hepatocytes as it contains no potentially toxic reagents.

One major limitation of the present study was the method of application of HSM to HCC. It did not seem practical to apply HSM intravenously. Transcatheter arterial chemoembolization is an established method for the treatment of HCC (23). Balloon occlusion of the hepatic artery is a novel technique (21). The balloon occlusion uses a micro balloon to obstruct the hepatic artery. HCC tissues are immersed with chemotherapeutic agents following the occlusion. HSM could be used to immerse HCC tissues instead of chemotherapeutic agents. With the occlusion technique, it is possible that applying HSM may be more practical.

In conclusion, HCC cells and HUVECs died in a medium without glucose or arginine, and supplemented with galactose and arginine, namely HSM. HSM might be useful for the harmless treatment of HCC as normal hepatocytes are able to survive in HSM

\section{References}

1. Zhu RX, Seto WK, Lai CL and Yuen MF: Epidemiology of hepatocellular carcinoma in the Asia-Pacific region. Gut Liver 10: 332-339, 2016.

2. Tomizawa M, Kondo F and Kondo Y: Growth patterns and interstitial invasion of small hepatocellular carcinoma. Pathol Int 45 352-358, 1995.

3. Lencioni R, Petruzzi P and Crocetti L: Chemoembolization of hepatocellular carcinoma. Semin Intervent Radiol 30: 3-11, 2013

4. Kim HY and Park JW: Clinical trials of combined molecular targeted therapy and locoregional therapy in hepatocellular carcinoma: Past, present and future. Liver Cancer 3: 9-17, 2014.
5. Raoul JL, Gilabert M and Piana G: How to define transarterial chemoembolization failure or refractoriness: A European perspective. Liver Cancer 3: 119-124, 2014.

6. Leffert HL and Paul D: Studies on primary cultures of differentiated fetal liver cells. J Cell Biol 52: 559-568, 1972.

7. Matsumoto K, Yamada K, Kohmura E, Kinoshita A and Hayakawa T: Role of pyruvate in ischaemia-like conditions on cultured neurons. Neurol Res 16: 460-464, 1994.

8. Ohira RH, Dipple KM, Zhang YH and McCabe ER: Human and murine glycerol kinase: Influence of exon 18 alternative splicing on function. Biochem Biophys Res Commun 331: 239-246, 2005.

9. Ai Y, Jenkins NA, Copeland NG, Gilbert DH, Bergsma DJ and Stambolian D: Mouse galactokinase: Isolation, characterization and location on chromosome 11. Genome Res 5: 53-59, 1995.

10. Phillips JW, Jones ME and Berry MN: Implications of the simultaneous occurrence of hepatic glycolysis from glucose and gluconeogenesis from glycerol. Eur J Biochem 269: 792-797, 2002

11. Sumida KD, Crandall SC, Chadha PL and Qureshi T: Hepatic gluconeogenic capacity from various precursors in young versus old rats. Metabolism 51: 876-880, 2002.

12. Wheatley DN, Scott L, Lamb J and Smith S: Single amino acid (arginine) restriction: Growth and death of cultured HeLa and human diploid fibroblasts. Cell Physiol Biochem 10: 37-55, 2000.

13. Walker V: Ammonia toxicity and its prevention in inherited defects of the urea cycle. Diabetes Obes Metab 11: 823-835, 2009.

14. Tomizawa $\mathrm{M}$, Toyama $\mathrm{Y}$, Ito $\mathrm{C}$, Toshimori $\mathrm{K}$, Iwase $\mathrm{K}$, Takiguchi M, Saisho H and Yokosuka O: Hepatoblast-like cells enriched from mouse embryonic stem cells in medium without glucose, pyruvate, arginine and tyrosine. Cell Tissue Res 333: 17-27, 2008

15. Tomizawa M, Shinozaki F, Sugiyama T, Yamamoto $\mathrm{S}$, Sueishi M and Yoshida T: Survival of primary human hepatocytes and death of induced pluripotent stem cells in media lacking glucose and arginine. PLoS One 8: e71897, 2013.

16. Nakamura T, Teramoto H, Tomita Y and Ichihara A: L-proline is an essential amino acid for hepatocyte growth in culture. Biochem Biophys Res Commun 122: 884-891, 1984.

17. Davies B and Fried M: The L19 ribosomal protein gene (RPL19): Gene organization, chromosomal mapping, and novel promoter region. Genomics 25: 372-380, 1995.

18. Tam S, Clavijo A, Engelhard EK and Thurmond MC: Fluorescence-based multiplex real-time RT-PCR arrays for the detection and serotype determination of foot-and-mouth disease virus. J Virol Methods 161: 183-191, 2009.

19. Simons AL, Mattson DM, Dornfeld K and Spitz DR: Glucose deprivation-induced metabolic oxidative stress and cancer therapy. J Cancer Res Ther 5 (Suppl 1): S2-S6, 2009.

20. Módis K, Gerő D, Stangl R, Rosero O, Szijártó A, Lotz G, Mohácsik P, Szoleczky P, Coletta C and Szabó C: Adenosine and inosine exert cytoprotective effects in an in vitro model of liver ischemia-reperfusion injury. Int $\mathrm{J}$ Mol Med 31: 437-446, 2013

21. Cai H, Jiang D, Qi F, Xu J, Yu L and Xiao Q: HRP-3 protects the hepatoma cells from glucose deprivation-induced apoptosis. Int J Clin Exp Pathol 8: 14383-14391, 2015

22. Feun L, You M, Wu CJ, Kuo MT, Wangpaichitr M, Spector S and Savaraj N: Arginine deprivation as a targeted therapy for cancer. Curr Pharm Des 14: 1049-1057, 2008

23. Ju W, Li S, Wang Z, Liu Y and Wang D: Decorin protects human hepatocma HepG2 cells against oxygen-glucose deprivation via modulating autophagy. Int J Clin Exp Med 8: $13347-13452,2015$ 